\title{
On Construction of Solutions of Evolutionary Nonlinear Schrödinger Equation
}

\author{
Andrey Melnikov \\ SunEdison, 501 Pearl Drive, St. Peters, MO 63376, USA \\ Correspondence should be addressed to Andrey Melnikov; andreymath@gmail.com
}

Received 1 May 2014; Accepted 30 June 2014; Published 7 October 2014

Academic Editor: Nikolai A. Kudryashov

Copyright ( 2014 Andrey Melnikov. This is an open access article distributed under the Creative Commons Attribution License, which permits unrestricted use, distribution, and reproduction in any medium, provided the original work is properly cited.

In this work we present an application of a theory of vessels to a solution of the evolutionary nonlinear Schrödinger (NLS) equation. The classes of functions for which the initial value problem is solvable rely on the existence of an analogue of the inverse scattering theory for the usual NLS equation. This approach is similar to the classical approach of Zakharov-Shabath for solving evolutionary NLS equation but has an advantage of simpler formulas and new techniques and notions to understand the solutions.

\section{Introduction and Background}

Solutions of the nonlinear Schrödinger (NLS) evolutionary equation play a special role in the theory of PDEs and in physics (optics and water waves). This equation can be defined as follows:

$$
i y_{t}+y_{x x}+2|y|^{2} y=0, \quad y(x, 0)=\beta(x),
$$

where $y=y(x, t)$ is a complex valued function of two real variables $x, t$ and $y(x, 0)=\beta(x)$ is the initial condition, defined on an interval $I \subseteq \mathbb{R}$. Notice that constant 2 used in this work can be replaced with arbitrary number by scaling of the variables. This equation has so-called integrability property, which enables the usage of the inverse scattering theory and Zakharov-Shabath system [1]. Additionally, there are also numerous numerical solutions of this equation, among which we can mention split-step (Fourier) method. In this work we are going to generalize the method of Zakharov-Shabath by introducing evolutionary NLS (regular) vessels. Although the setting we choose to work with involves bounded operators only and Hilbert space techniques, there are many classes of analytic solutions of the NLS equation obtained. It is worth noticing that one can also generalize this theory to unbounded operators (as it was done in [2] for the SturmLiuoville differential equation).

We assume from the beginning that the initial value $\beta(x)$ is analytic on $I \subseteq \mathbb{R}$ function and moreover arises from a regular NLS vessel. From a recent work of the author [3] it follows that in a similar way to another completely integrable PDE, called Korteweg-de Vries, every analytic on $I$ function can be presented using a vessel and, as a result, the evolutionary NLS equation with this initial value is solvable. In this work we show examples of how to construct different $\beta(x)$, which have a bounded spectrum, a spectrum on a bounded, continuous curve, or an infinite discrete spectrum. The problem of constructing of a vessel for a given $\beta(x)$ is identical to the inverse scattering problem of the NLS equation. To emphasize this point let us briefly discuss how the construction of the solution of (1) is performed starting from the spectral data encoded in a $2 \times 2$ matrix-function $S(\lambda)$ which is realized [4] in the following manner:

$$
S(\lambda)=I-B_{0}^{*} \mathbb{X}_{0}^{-1}(\lambda I-A)^{-1} B_{0} .
$$

Here $\mathscr{H}$ is an auxiliary Hilbert space, on which there are defined bounded operators $A, \mathbb{X}_{0}$ and the operator $B_{0}: \mathbb{C}^{2} \rightarrow$ $\mathscr{H}$. We also assume that

$$
\mathbb{X}_{0}^{*}=\mathbb{X}_{0}, \quad A \mathbb{X}_{0}+\mathbb{X}_{0} A^{*}+B_{0} B_{0}^{*}=0
$$

Then solving for $B(x)$ in $(13) B^{\prime}(x)=-A B(x) \sigma_{2}$, for $\sigma_{2}=(1 / 2)\left[\begin{array}{cc}1 & 0 \\ 0 & -1\end{array}\right]$ and defining next $\mathbb{X}(x)=\mathbb{X}_{0}+$ $\int_{x_{0}}^{x} B(y) \sigma_{2} B^{*}(y) d y$ we will obtain that the function

$$
\beta(x)=\left[\begin{array}{ll}
1 & 0
\end{array}\right] B^{*} \mathbb{X}^{-1} B\left[\begin{array}{l}
0 \\
1
\end{array}\right]
$$


is analytic (Theorem 6) on an interval, where $\mathbb{X}(x)$ is invertible. On the other hand, $\mathbb{X}(x)$ is invertible on an interval $I$ including $x_{0}$ because $\mathbb{X}_{0}$ is such. Indeed, at the definition of $\mathbb{X}(x)$, we add to $\mathbb{X}_{0}$ a bounded operator with bounded growth. Moreover, it turns out (Theorem 5) that the function

$$
S(\lambda, x)=I-B^{*}(x) \mathbb{X}^{-1}(x)(\lambda I-A)^{-1} B(x)
$$

maps solutions $u(\lambda, x)$ of the trivial NLS equation (24)

$$
\frac{\partial}{\partial x} u(\lambda, x)-(-i \lambda) \sigma_{2} u(\lambda, x)=0
$$

to solutions $y(\lambda, x)=S(\lambda, x) u(\lambda, x)$ of the NLS equation (25)

$$
\frac{\partial}{\partial x} y(\lambda, x)-(-i \lambda) \sigma_{2}(\lambda, x)+\left[\begin{array}{cc}
0 & \beta(x) \\
-\beta^{*}(x) & 0
\end{array}\right] u(x, \lambda)=0 .
$$

In order to obtain a solution of the evolutionary NLS (1), we evolve the operator $B$ with respect to $t$ as follows:

$$
\begin{gathered}
\frac{\partial}{\partial t} B(x, t)=i A \frac{\partial}{\partial x} B(x, t)=i A\left(-A B(x, t) \sigma_{2}\right), \\
B(x, 0)=B(x)
\end{gathered}
$$

and redefine

$$
\begin{aligned}
\mathbb{X}(x, t)= & \mathbb{X}(x) \\
& +\int_{0}^{t}\left[i A B(x, s) \sigma_{2} B^{*}(x, s)\right. \\
& \left.-i B(x, s) \sigma_{2} B^{*}(x, s) A^{*}\right] d s .
\end{aligned}
$$

One of the most interesting results of this paper is Theorem 17 , where we prove that the new

$$
\beta(x, t)=\left[\begin{array}{ll}
1 & 0
\end{array}\right] B^{*}(x, t) \mathbb{X}^{-1}(x, t) B(x, t)\left[\begin{array}{l}
0 \\
1
\end{array}\right]
$$

satisfies (1) and coincides with $\beta(x)$ for $t=0$, proving the existence of solutions for (1) with initial value.

The formulas presented here enable us also to explicitly perform this construction for some basic and important cases. We show how to construct a vessel, for which the spectrum of $A$ lies on a curve $\Gamma$ (Section 3.2 for $\beta(x)$ and Section 5.2 for $\beta(x, t)$ ), with a discrete set (Section 3.3 for $\beta(x)$ and Section 5.3 for $\beta(x, t))$. We also discuss the general construction in Sections 3.1 and 5.1. Finally we present constructions of the Solitons in Section 5.4. Much of the results presented here were originated in the work of the author and collaborators [5-8]. As we pointed out, as this paper was being processed for the publication, the result of solving the KdV equation with analytic initial condition was accepted for publication in the Journal of Math. Physics. [3], showing that a similar result is correct for the NLS equation too.

\section{Definition of a Regular NLS Vessel and Its Properties}

We define first parameters, which will be frequently used in the sequel. Another choice of these parameters generates solutions of the Sturm-Liuoville differential equation and the Kortweg-de-Vries equation (see [2,9] for details). A similar result was presented for the setting canonical systems and their evolution [10].

Definition 1. NLS vessel parameters are defined as follows:

$$
\sigma_{1}=\left[\begin{array}{ll}
1 & 0 \\
0 & 1
\end{array}\right], \quad \sigma_{2}=\frac{1}{2}\left[\begin{array}{cc}
1 & 0 \\
0 & -1
\end{array}\right], \quad \gamma=\left[\begin{array}{ll}
0 & 0 \\
0 & 0
\end{array}\right] .
$$

Definition 2. An NLS regular vessel is a collection of operators and spaces:

$$
\mathfrak{V}_{\mathrm{NLS}}=\left(A, B(x), \mathbb{X}(x) ; \sigma_{1}, \sigma_{2}, \gamma, \gamma_{*}(x) ; \mathscr{H}, \mathbb{C}^{2}, I\right),
$$

where $\sigma_{1}, \sigma_{2}$, and $\gamma$ are the NLS vessel parameters and $I$ is a closed interval. The operators $A, \mathbb{X}(x): \mathscr{H} \rightarrow \mathscr{H}, B(x)$ : $\mathbb{C}^{2} \rightarrow \mathscr{H}$ are bounded operators for all $x \in I$. The operator $\mathbb{X}(x)$ is invertible for all $x \in I$. The operators are subject to the following vessel conditions:

$$
\begin{gathered}
0=\frac{d}{d x}(B(x))+A B(x) \sigma_{2} \\
A \mathbb{X}(x)+\mathbb{X}(x) A^{*}+B(x) B(x)^{*}=0 \\
\frac{d}{d x} \mathbb{X}(x)=B(x) \sigma_{2} B(x)^{*} \\
\gamma_{*}(x)=\sigma_{2} B(x)^{*} \mathbb{X}^{-1}(x) B(x)-B(x)^{*} \mathbb{X}^{-1}(x) B(x) \sigma_{2} \\
\mathbb{X}^{*}(x)=\mathbb{X}(x) .
\end{gathered}
$$

For each NLS vessel there exist three notions, which play a significant role in research.

Definition 3. Suppose that we are given an NLS vessel $\mathfrak{V}_{\mathrm{NLS}}$. Then its transfer function $S(\lambda, x)$, the tau-functions $\tau(x)$, and the beta-function $\beta(x)$ are defined as follows:

$$
\begin{gathered}
S(\lambda, x)=I-B(x)^{*} \mathbb{X}^{-1}(x)(\lambda I-A)^{-1} B(x), \\
\tau(x)=\operatorname{det}\left(\mathbb{X}^{-1}\left(x_{0}\right) \mathbb{X}(x)\right), \\
\beta(x)=\left[\begin{array}{ll}
1 & 0
\end{array}\right] \gamma_{*}(x)\left[\begin{array}{l}
0 \\
1
\end{array}\right]=\left[\begin{array}{ll}
1 & 0
\end{array}\right] B^{*}(x) \mathbb{X}^{-1}(x) B(x)\left[\begin{array}{l}
0 \\
1
\end{array}\right] .
\end{gathered}
$$

The definition of $\beta(x)$ may be considered as excessive, because actually the matrix-function $\gamma_{*}(x)$ turns to be

$$
\gamma_{*}(x)=\left[\begin{array}{cc}
0 & \beta(x) \\
-\beta^{*}(x) & 0
\end{array}\right],
$$

using the self-adjointness of $\mathbb{X}(x)$. Still, we will use these two notions extensively, so we have defined both of them. 
Notice that $S(\lambda, x)$ is a $2 \times 2$ matrix-function, whose poles and singularities with respect to $\lambda$ are determined by the operator $A$ only. Since all the involved operators, appearing at the definition of $S(\lambda, x)$, are bounded, we can see that $S(\lambda, x)$ is analytic in $\lambda$ for all $x \in I$ with value $I$ (=identity $2 \times 2$ matrix) there. As a result, we can consider its Taylor series

$$
\begin{aligned}
S(\lambda, x) & =I-B^{*}(x) \mathbb{X}^{-1}(x)(\lambda I-A)^{-1} B(x) \sigma_{1} \\
& =I-\sum_{i=0}^{\infty} \frac{H_{i}(x)}{\lambda^{i+1}} \sigma_{1},
\end{aligned}
$$

which is convergent at least for $\lambda>\|A\|$. Thus we define its (Markov) moments as follows.

Definition 4. $n$th moment of the vessel $\mathfrak{V}_{\mathrm{NLS}}$ is

$$
H_{n}(x)=B^{*}(x) \mathbb{X}^{-1}(x) A^{n} B(x) .
$$

We will present in the next section basic properties of an NLS vessel $\mathfrak{V}_{\mathrm{NLS}}$ by exploring all the objects (the transfer, the tau, the beta functions, and the moments). We will also see that there is a standard technique for construction of such vessels.

2.1. The Transfer and the Tau-Function of an NLS Vessel. The main reason to consider NLS vessel is Theorem 5, whose proof we present in full detail, and also it appeared in different settings in [5,11-14]. It turns out that we can see an NLS vessel as a Bäcklund transformation of the trivial NLS equation to a more complicated one. More precisely, the transfer function $S(\lambda, x)$ of such a vessel maps solutions of the so-called input $L D E$ with the spectral parameter $\lambda$

$$
\left[\lambda \sigma_{2}-\sigma_{1} \frac{\partial}{\partial x}+\gamma\right] u(\lambda, x)=0
$$

to solutions of the output $L D E$ with the same spectral parameter

$$
\left[\lambda \sigma_{2}-\sigma_{1} \frac{\partial}{\partial x}+\gamma_{*}\right] y(\lambda, x)=0
$$

As a result the following differential equation holds:

$$
S_{x}^{\prime}=\sigma_{1}^{-1}\left(\sigma_{2} \lambda+\gamma_{*}\right) S-S \sigma_{1}^{-1}\left(\sigma_{2} \lambda+\gamma\right) .
$$

Theorem 5 (vessel as a Bäcklund transformation). Suppose that $\mathfrak{V}_{N L S}$ is an NLS vessel, defined in (12), and suppose that $u(x, \lambda)=\left[\begin{array}{l}u_{1}(\lambda, x) \\ u_{2}(\lambda, x)\end{array}\right]$ is a solution of the input LDE (24)

$$
\lambda \sigma_{2} u(\lambda, x)-\sigma_{1} \frac{\partial}{\partial x} u(\lambda, x)+\gamma u(\lambda, x)=0
$$

then $y(\lambda, x)=S(\lambda, x) u(\lambda)$ is a solution of the output $L D E(25)$

$$
\lambda \sigma_{2} y(\lambda, x)-\sigma_{1} \frac{\partial}{\partial x} y(\lambda, x)+\gamma_{*}(x) y(\lambda, x)=0 .
$$

Proof. We plug in the expression

$$
\begin{aligned}
y(\lambda, x) & =S(\lambda, x) u(\lambda) \\
& =\left(I-B^{*}(x) \mathbb{X}^{-1}(x)(\lambda I-A)^{-1} B(x) \sigma_{1}\right) u(\lambda, x)
\end{aligned}
$$

into (25) for all $\lambda \notin \operatorname{spec}(A)$. Let us denote $G(\lambda, x)=$ $B^{*}(x) \mathbb{X}^{-1}(x)(\lambda I-A)^{-1} B(x)$; then, for all $x \in I$ and $\lambda \notin$ $\operatorname{spec}(A)$

$$
\begin{aligned}
\lambda \sigma_{2} y & (\lambda, x)-\sigma_{1} \frac{\partial}{\partial x} y(\lambda, x)+\gamma_{*}(x) y(\lambda, x) \\
= & \lambda \sigma_{2}\left[\left(I-G(\lambda, x) \sigma_{1}\right) u(\lambda, x)\right] \\
& -\sigma_{1} \frac{\partial}{\partial x}\left[\left(I-G(\lambda, x) \sigma_{1}\right) u(\lambda, x)\right] \\
& +\gamma_{*}(x)\left(I-G(\lambda, x) \sigma_{1} u\right)(\lambda, x) \\
= & \lambda \sigma_{2} u(\lambda, x)-\lambda \sigma_{2} G(\lambda, x) \sigma_{1} u(\lambda, x) \\
& -\sigma_{1} \frac{\partial}{\partial x} u(\lambda, x)+\sigma_{1} \frac{\partial}{\partial x}[G(\lambda, x)] \sigma_{1} u(\lambda, x) \\
& -\sigma_{1} G(\lambda, x) \sigma_{1} \frac{\partial}{\partial x} u(\lambda, x)+\gamma_{*}(x) u(\lambda, x) \\
& -\gamma_{*}(x) G(\lambda, x) \sigma_{1} u(\lambda, x) \\
= & \mathrm{using}(24) \\
= & -\lambda \sigma_{2} G(\lambda, x) \sigma_{1} u(\lambda, x)+\sigma_{1} \frac{\partial}{\partial x}[G(\lambda, x)] \sigma_{1} u(\lambda, x) \\
& -\sigma_{1} G(\lambda, x) \sigma_{1} \frac{\partial}{\partial x} u(\lambda, x) \\
& \left(\gamma_{*}(x)-\gamma\right) u(\lambda, x)-\gamma_{*}(x) G(\lambda, x) \sigma_{1} u(\lambda, x)
\end{aligned}
$$

Let us differentiate the expression $\sigma_{1} G(\lambda, x) \sigma_{1}$ using formulas (13) and (15):

$$
\begin{aligned}
& \sigma_{1} \frac{\partial}{\partial x} G(\lambda, x) \sigma_{1} \\
&=\frac{\partial}{\partial x}\left[\sigma_{1} B^{*}(x) \mathbb{X}^{-1}(x)(\lambda I-A)^{-1} B(x) \sigma_{1}\right] \\
&=\left(-\sigma_{2} B^{*}(x) A^{*}-\gamma B^{*}(x)\right) \mathbb{X}^{-1}(x)(\lambda I-A)^{-1} B(x) \sigma_{1} \\
& \quad-\sigma_{1} B^{*}(x) \mathbb{X}^{-1}(x) B(x) \sigma_{2} G(\lambda, x) \sigma_{1} \\
& \quad+\sigma_{1} B^{*}(x) \mathbb{X}^{-1}(x)(\lambda I-A)^{-1}\left(-A B(x) \sigma_{2}-B(x) \gamma\right) \\
&=-\sigma_{2} B^{*}(x) A^{*} \mathbb{X}^{-1}(x)(\lambda I-A)^{-1} B(x) \sigma_{1} \\
& \quad-\gamma G(\lambda, x) \sigma_{1}-\sigma_{1} B^{*}(x) \mathbb{X}^{-1}(x) B(x) \sigma_{2} G(\lambda, x) \sigma_{1} \\
& \quad-\sigma_{1} B^{*}(x) \mathbb{X}^{-1}(x)(\lambda I-A)^{-1} A B(x) \sigma_{2}-\sigma_{1} G(\lambda, x) \gamma \\
&= \mathrm{u} \operatorname{sing}(14) \text { for } A^{*} \mathbb{X}^{-1}(x)
\end{aligned}
$$




$$
\begin{aligned}
= & -\sigma_{2} B^{*}(x)\left(-\mathbb{X}^{-1}(x) A-\mathbb{X}^{-1}(x) B(x) \sigma_{1} B^{*}(x) \mathbb{X}^{-1}(x)\right) \\
& \times(\lambda I-A)^{-1} B(x) \sigma_{1} \\
& -\gamma G(\lambda, x) \sigma_{1}-\sigma_{1} B^{*}(x) \mathbb{X}^{-1}(x) B(x) \sigma_{2} G(\lambda, x) \sigma_{1} \\
& -\sigma_{1} B^{*}(x) \mathbb{X}^{-1}(x)(\lambda I-A)^{-1} A B(x) \sigma_{2}-\sigma_{1} G(\lambda, x) \gamma \\
= & \sigma_{2} B^{*}(x) \mathbb{X}^{-1}(x)(A \pm \lambda I)(\lambda I-A)^{-1} B(x) \sigma_{1} \\
& +\sigma_{2} B^{*}(x) \mathbb{X}^{-1}(x) B(x) \sigma_{1} G(\lambda, x) \sigma_{1} \\
& -\gamma G(\lambda, x) \sigma_{1}-\sigma_{1} B^{*}(x) \mathbb{X}^{-1}(x) B(x) \sigma_{2} G(\lambda, x) \sigma_{1} \\
& -\sigma_{1} B^{*}(x) \mathbb{X}^{-1}(x)(\lambda I-A)^{-1}(A \pm \lambda I) B(x) \sigma_{2} \\
& -\sigma_{1} G(\lambda, x) \gamma \\
= & -\sigma_{2} B^{*}(x) \mathbb{X}^{-1}(x) B(x) \sigma_{1}+\lambda \sigma_{2} G(\lambda, x) \sigma_{1} \\
& +\sigma_{2} B^{*}(x) \mathbb{X}^{-1}(x) B(x) \sigma_{1} G(\lambda, x) \sigma_{1} \\
& -\gamma G(\lambda, x) \sigma_{1}-\sigma_{1} B^{*}(x) \mathbb{X}^{-1}(x) B(x) \sigma_{2} G(\lambda, x) \sigma_{1} \\
& +\sigma_{1} B^{*}(x) \mathbb{X}^{-1}(x) B(x) \sigma_{2} \\
& -\lambda \sigma_{1} G(\lambda, x) \sigma_{2}-\sigma_{1} G(\lambda, x) \gamma .
\end{aligned}
$$

Plugging this expression back into (25) developed earlier and performing some obvious cancellations, we will obtain

$$
\begin{aligned}
\lambda \sigma_{2} y & (\lambda, x)-\sigma_{1} \frac{\partial}{\partial x} y(\lambda, x)+\gamma_{*}(x) y(\lambda, x) \\
=- & \lambda \sigma_{2} G(\lambda, x) \sigma_{1} u(\lambda, x)+\sigma_{1} \frac{\partial}{\partial x}[G(\lambda, x)] \sigma_{1} u(\lambda, x) \\
- & \sigma_{1} G(\lambda, x) \sigma_{1} \frac{\partial}{\partial x} u(\lambda, x) \\
+ & \left(\gamma_{*}(x)-\gamma\right) u(\lambda, x)-\gamma_{*}(x) G(\lambda, x) \sigma_{1} u(\lambda, x) \\
= & -\sigma_{2} B^{*}(x) \mathbb{X}^{-1}(x) B(x) \sigma_{1} \\
& \left.+\sigma_{1} B^{*}(x) \mathbb{X}^{-1}(x) B(x) \sigma_{1}+\gamma_{*}(x)-\gamma\right) u(\lambda, x) \\
+ & \left(\sigma_{2} B^{*}(x) \mathbb{X}^{-1}(x) B(x) \sigma_{1}-\sigma_{1} B^{*}(x) \mathbb{X}^{-1}(x) B(x) \sigma_{1}\right. \\
& \left.+\gamma_{*}(x)-\gamma\right) G(\lambda, x) \sigma_{1} u(\lambda, x)+\sigma_{1} G(\lambda, x) \\
\times & \left(\sigma_{1} \frac{\partial}{\partial x} u(\lambda, x)-\lambda \sigma_{2} u(\lambda, x)+\gamma u(\lambda, x)\right)=0,
\end{aligned}
$$

using linkage condition (16) and the differential equation (24).

Let us present next the significance (and well-definedness) of the tau-function $\tau(x)$, defined in (19). Using vessel condition (15) $\mathbb{X}(x)$ has the formula

$$
\mathbb{X}(x)=\mathbb{X}\left(x_{0}\right)+\int_{0}^{x} B^{*}(y) \sigma_{2} B(y) d y,
$$

and as a result

$$
\mathbb{X}^{-1}\left(x_{0}\right) \mathbb{X}(x)=I+\mathbb{X}^{-1}\left(x_{0}\right) \int_{x_{0}}^{x} B^{*}(y) \sigma_{2} B(y) d y .
$$

Since $\sigma_{2}$ has rank 2, this expression is of the form $I+T$, for a trace-class operator $T$, and since $\mathbb{X}_{0}$ is an invertible operator, there exists a nontrivial interval (of length at least $\left.1 /\left\|\mathbb{X}_{0}^{-1}\right\|\right)$ on which $\mathbb{X}(x)$ and $\tau(x)$ are defined. Recall [15] that a function $F(x)$ from $(a, b)$ into the group $G$ (the set of bounded invertible operators on $H$ of the form $I+T$, for a trace-class operator $T$ ) is said to be differentiable if $F(x)-I$ is differentiable as a map into the trace-class operators. In our case,

$$
\begin{aligned}
\frac{d}{d x}\left(\mathbb{X}^{-1}\left(x_{0}\right) \mathbb{X}(x)\right) & =\mathbb{X}^{-1}\left(x_{0}\right) \frac{d}{d x} \mathbb{X}(x) \\
& =\mathbb{X}^{-1}\left(x_{0}\right) B(x) \sigma_{2} B^{*}(x)
\end{aligned}
$$

exists in trace-class norm. Israel Gohberg and Mark Krein $\left[15\right.$, formula 1.14 on $\mathrm{p}$. 163] proved that if $\mathbb{X}^{-1}\left(x_{0}\right) \mathbb{X}(x)$ is a differentiable function into $G$, then $\tau(x)=\operatorname{sp}\left(\mathbb{X}^{-1}\left(x_{0}\right) \mathbb{X}(x)\right)$ (sp stands for the trace in the infinite dimensional space) is a differentiable map into $\mathbb{C}^{*}$ with

$$
\begin{aligned}
\frac{\tau^{\prime}}{\tau} & =\operatorname{sp}\left(\left(\mathbb{X}^{-1}\left(x_{0}\right) \mathbb{X}(x)\right)^{-1} \frac{d}{d x}\left(\mathbb{X}^{-1}\left(x_{0}\right) \mathbb{X}(x)\right)\right) \\
& =\operatorname{sp}\left(\mathbb{X}(x)^{\prime} \mathbb{X}^{-1}(x)\right) \\
& =\operatorname{sp}\left(B(x) \sigma_{2} B^{*}(x) \mathbb{X}^{-1}(x)\right) \\
& =\operatorname{tr}\left(\sigma_{2} B^{*}(x) \mathbb{X}^{-1}(x) B(x)\right) .
\end{aligned}
$$

The most important question, related to this theory, is what classes of $\beta(x)$ are obtained. This question is answered in the next theorem.

Theorem 6. Suppose that $\mathfrak{B}_{N L S}$ is an NLS vessel, defined in (12). Then the function $\beta(x)$ is analytic on the interval I.

Proof. Notice that from formula (13) it follows the operator $B(x)$ is analytic in $x$. Since $\mathbb{X}(x)$ is invertible on $I$, the operator $\mathbb{X}^{-1}(x)$ is also analytic in $x$ using the formula

$$
\frac{d}{d x} \mathbb{X}^{-1}(x)=-\mathbb{X}^{-1}(x) B(x) \sigma_{2} B^{*}(x) \mathbb{X}^{-1}(x) .
$$

Thus $\beta(x)$, defined by (20), is analytic on $I$.

Theorem 7 (permanency conditions). Suppose that we are given an NLS regular vessel $\mathfrak{B}_{N L S}$; then

(1) if the Lyapunov equation (14) holds for a fixed $x_{0} \in I$, then it holds for all $x \in I$,

(2) if $S^{*}\left(-\bar{\lambda}, x_{0}\right) \sigma_{1} S\left(\lambda, x_{0}\right)=\sigma_{1}$, then

$$
S^{*}(-\bar{\lambda}, x) \sigma_{1} S(\lambda, x)=\sigma_{1},
$$

for all $x \in I$, 
(3) $\operatorname{det} S(\lambda, x)=\operatorname{det} S\left(\lambda, x_{0}\right)$ for all $x_{0}, x \in I$ and all points of $\lambda$-analyticity of $S(\lambda, x)$.

Proof. Differentiating Lyapunov equation and using vessel conditions (13) and (15) we will obtain that

$$
\begin{aligned}
A \frac{d}{d x} \mathbb{X}(x)+\frac{d}{d x} \mathbb{X}(x) A^{*}+\frac{d}{d x}[B(x)] B^{*}(x) \\
\quad+B(x) \frac{d}{d x} B^{*}(x) \\
=A B(x) \sigma_{2} B^{*}(x)+B(x) \sigma_{2} B^{*}(x) A^{*} \\
\quad+\left(-A B(x) \sigma_{2}\right) B^{*}(x)+B(x)\left(-\sigma_{2} B^{*} A^{*}\right)=0
\end{aligned}
$$

from where the permanency of the Lyapunov equation follows. Similarly, differentiating $S^{*}(-\bar{\lambda}, x) \sigma_{1} S(\lambda, x)$ we will obtain zero and the permanency of (38) follows. For the last statement, using (26) we calculate for $\lambda \notin \operatorname{spec}(A)$

$$
\begin{aligned}
& \frac{(\partial / \partial x) \operatorname{det} S(\lambda, x)}{\operatorname{det} S(\lambda, x)} \\
& =\operatorname{tr}\left(S^{-1}(\lambda, x) \frac{\partial}{\partial x} S(\lambda, x)\right) \\
& =\operatorname{tr}\left(S^{-1}(\lambda, x)\left[\left(\sigma_{2} \lambda+\gamma_{*}(x)\right) S(\lambda, x)-S(\lambda, x)\left(\sigma_{2} \lambda+\gamma\right)\right]\right) \\
& =\operatorname{tr}\left(\sigma_{2} \lambda+\gamma_{*}(x)-\left(\sigma_{2} \lambda+\gamma\right)\right) \\
& =\operatorname{tr}\left(\gamma_{*}(x)-\gamma(x)\right) \\
& =\operatorname{tr}\left(B^{*}(x) \mathbb{X}^{-1}(x) B(x) \sigma_{2}-\sigma_{2} B^{*}(x) \mathbb{X}^{-1}(x) B(x)\right)=0 .
\end{aligned}
$$

We will not be using the last property in this statement, but we find it interesting by itself.

2.2. Moments. The following properties of the moments $H(x)$ of an NLS vessel are immediate from their definition as the coefficients of $1 / \lambda^{n+1}$ at the Taylor series of $S(\lambda, x)$.

Theorem 8. Let $\mathfrak{V}_{N L S}$ be an NLS vessel. Then its moments satisfy the following equations:

$$
\begin{gathered}
\sigma_{1}^{-1} \sigma_{2} H_{n+1}-H_{n+1} \sigma_{2} \sigma_{1}^{-1}=\frac{d}{d x} H_{n}-\sigma_{1}^{-1} \gamma_{*} H_{n}+H_{n} \gamma \sigma_{1}^{-1}, \\
H_{n+1}+(-1)^{n} H_{n+1}^{*}=\sum_{j=0}^{n}(-1)^{j+1} H_{n-j} \sigma_{1} H_{j}^{*} .
\end{gathered}
$$

Proof. Plugging the Taylor expansion formula into (26) and equating the coefficients of $1 / \lambda^{n=1}$ we will obtain the first formula (41). The second formula (42) is obtained in the same manner from (42).
It turns out that using only the differential equations (41) one can create a recursive formula for the entries $H_{n}=$ $\left[\begin{array}{ll}H_{n}^{11} & H_{n}^{12} \\ H_{n}^{21} & H_{n}^{22}\end{array}\right]$ as follows:

$$
\begin{aligned}
H_{n}^{21} & =-\frac{d}{d x}\left(H_{n-1}^{21}\right)-\beta^{*} H_{n-1}^{11}, \\
H_{n}^{12} & =\frac{d}{d x}\left(H_{n-1}^{12}\right)-\beta H_{n-1}^{22}, \\
\frac{d}{d x}\left(H_{n}^{11}\right) & =\beta H_{n}^{21} \\
\frac{d}{d x}\left(H_{n}^{22}\right) & =-\beta^{*} H_{n}^{12},
\end{aligned}
$$

while the first moment $H_{0}$ is found from linkage condition (16)

$$
H_{0}=\left[\begin{array}{cc}
H_{0}^{11} & \beta \\
\beta^{*} & H_{0}^{22}
\end{array}\right],
$$

and the entries $H_{0}^{11}, H_{0}^{22}$ are found using two last equations of (43):

$$
\begin{aligned}
& \frac{d}{d x}\left(H_{0}^{11}\right)=\beta H_{0}^{21}=\beta \beta^{*}, \\
& \frac{d}{d x}\left(H_{0}^{22}\right)=-\beta^{*} H_{0}^{12}=-\beta^{*} \beta .
\end{aligned}
$$

As a result, we obtain that the formula for the tau-function (36) becomes

$$
\begin{aligned}
\frac{\tau^{\prime}}{\tau} & =\operatorname{tr}\left(\sigma_{2} B^{*}(x) \mathbb{X}^{-1}(x) B(x)\right)=\operatorname{tr}\left(\sigma_{2} H_{0}\right) \\
& =\frac{1}{2}\left(H_{0}^{11}-H_{0}^{22}\right) \\
& =\frac{1}{2}\left(H_{0}^{11}(0)-H_{0}^{22}(0)\right)+\int_{x_{0}}^{x}|\beta(y)|^{2} d y .
\end{aligned}
$$

For example, if we choose the initial parameters $H_{0}^{11}(0)$, $H_{0}^{22}(0)$ to be equal, we will obtain that under normalization $\tau\left(x_{0}\right)=1$

$$
\tau(x)=e^{\int_{x_{0}}^{x} \int_{x_{0}}^{t}|\beta(y)|^{2} d y d t} .
$$

\section{Examples of Constructions of Regular NLS Vessels}

3.1. Construction of an NLS Vessel from a Realized Function. Construction of an NLS vessel from the scattering data (initial condition $\left.S\left(x_{0}, \lambda\right)\right)$ can be performed as follows. Suppose that the function $S\left(\lambda, x_{0}\right)$ is realized [4] as follows:

$$
S\left(x_{0}, \lambda\right)=I-B_{0} \mathbb{X}_{0}^{-1}(\lambda I-A)^{-1} B_{0} \sigma_{1},
$$

satisfying additionally $A \mathbb{X}_{0}+\mathbb{X}_{0} A^{*}+B_{0} B_{0}^{*}=0$ and $\mathbb{X}_{0}^{*}=\mathbb{X}_{0}$. These two conditions are required by the 
permanency conditions (Theorem 7) and will hold for all $x$ by the construction. Then define $B(x)$ as the unique solution of (13) with initial value $B_{0}$ :

$$
0=\frac{d}{d x}(B(x))+A B(x) \sigma_{2}, \quad B(0)=B_{0}
$$

Then define

$$
\mathbb{X}(x)=\mathbb{X}_{0}+\int_{x_{0}}^{x} B(y) \sigma_{2} B^{*}(y) d y,
$$

and define $\gamma_{*}(x)$ (and hence $\beta(x)$ ) using (16). It is straightforward to check that all vessel conditions hold.

Theorem 9. Suppose that we are given a function

$$
S\left(x_{0}, \lambda\right)=I-B_{0} \mathbb{X}_{0}^{-1}(\lambda I-A)^{-1} B_{0}
$$

where using an auxiliary Hilbert space $\mathscr{H}$ the operators act as follows

$$
A, \mathbb{X}_{0}: \mathscr{H} \longrightarrow \mathscr{H}, \quad B_{0}: \mathbb{C}^{2} \longrightarrow \mathscr{H}
$$

and satisfy $A \mathbb{X}_{0}+\mathbb{X}_{0} A^{*}+B_{0} B_{0}^{*}=0$ and $\mathbb{X}_{0}^{*}=\mathbb{X}_{0}$. Define $a$ collection (12) using the formulas (49), (50), and (16)

$$
\mathfrak{V}_{N L S}=\left(A, B(x), \mathbb{X}(x) ; \sigma_{1}, \sigma_{2}, \gamma, \gamma_{*}(x) ; \mathscr{H}, \mathbb{C}^{2}\right) ;
$$

then this collection is an NLS regular vessel existing on an interval I on which the operator $\mathbb{X}(x)$ is invertible.

We show two special examples, arising from this construction for special cases of the choice of $\mathscr{H}$.

3.2. Construction of a Regular NLS Vessel with the Spectrum on a Curve $\Gamma$. Let us fix a bounded continuous curve $\Gamma=\{\mu(t) \mid$ $t \in[a, b]\}$ (i.e., $\mu(t)$ is continuous) and define $\mathscr{H}=L^{2}(\Gamma)=$ $\left\{\left.f(\mu)\left|\int_{a}^{b}\right| f(\mu(t))\right|^{2} d t<\infty\right\}$. We suppose without loss of generality that $x_{0}=0$ and we construct a vessel, existing on an interval $I$ including zero.

Let $A=2 \mu$; that is, it is a bounded operator acting within $L^{2}(\Gamma)$. Solving differential equation (13) we find that

$$
B(x)=\left[e^{-\mu x} b_{1}(\mu) e^{\mu x} b_{2}(\mu)\right], \quad b_{1}(\mu), b_{2}(\mu) \in \mathscr{H} .
$$

Notice that the adjoint $B^{*}(x): \mathscr{H} \rightarrow \mathbb{C}^{2}$ is defined as follows:

$$
B^{*}(x) f(\mu)=\int_{a}^{b}\left[\begin{array}{c}
e^{-\bar{\mu}(t) x} \bar{b}_{1}(\mu(t)) \\
e^{\bar{\mu}(t) x} \bar{b}_{2}(\mu(t))
\end{array}\right] f(\mu(t)) d t .
$$

It is a well-defined operator, because by the Cauchy-Schwartz inequality the integrals are finite.

It turns out that the operator $\mathbb{X}(x)$ can be also explicitly defined as follows for any $f \in \mathscr{H}$. Notice that $\mathbb{X}(x) f$ is a new function at $\mathscr{H}$, for which we present its value at the point $\mu=$ $\mu(t) \in \Gamma$ :

$$
\begin{aligned}
&(\mathbb{X}(x) f)(\mu) \\
&=-\frac{1}{2} \int_{a}^{b}\left[\frac{b_{1}(\mu) \bar{b}_{1}(\mu(s)) e^{-(\mu+\bar{\mu}(s)) x}}{\mu+\bar{\mu}(s)}\right. \\
&\left.+\frac{b_{2}(\mu) \bar{b}_{2}(\mu(s)) e^{(\mu+\bar{\mu}(s)) x}}{\mu+\bar{\mu}(s)}\right] f(\mu(s)) d s .
\end{aligned}
$$

It is almost immediate that such an operator satisfies conditions (14) and (15). For example, for the Lyapunov equation, the expression $(A \mathbb{X}(x) f)(\mu)+\left(\mathbb{X}(x) A^{*} f\right)(\mu)$ is obtained by multiplying the expression under the integral in $(\mathbb{X}(x) f)(\mu)$ by $2(\mu+\bar{\mu}(s))$. When it is canceled with the denominator, we obtain $\left(-B(x) \sigma_{2} B^{*}(x) f\right)(\mu)$. Similarly, differentiating $(\mathbb{X}(x) f)(\mu)$ we cancel the denominator and switch the sign of the first term, so that (15) holds. There is only one problem, arising from the zero of the denominator $\mu(t)+\bar{\mu}(s)=0$. It can be overcome by requiring that either $\Gamma \cap\left(-\Gamma^{*}\right)=\emptyset$ or $b_{1}, b_{2}$ are Hölder functions and $b_{1}(\mu(t)) \bar{b}_{1}(\mu(s))+b_{2}(\mu(t)) \bar{b}_{2}(\mu(s))=0$, whenever $\mu(t)+\bar{\mu}(s)=0$ (so that the zero of the denominator is canceled by the numerator).

We will make the following assumption on the curve to simplify arguments.

Definition 10. Suppose that $\Gamma \cap\left(-\Gamma^{*}\right)=\emptyset$, where $-\Gamma^{*}=$ $\{-\bar{\mu}(t) \mid t \in[a, b]\}$.

Then investigating the formula for $\mathbb{X}(x)$ we find that for each $f \in \mathscr{H}$

$$
\begin{aligned}
(\mathbb{X}(x) f)(\mu) & \\
= & -\frac{b_{1}(\mu) e^{-\mu x}}{2} \int_{a}^{b} \frac{\bar{b}_{1}(\mu(s)) e^{-\bar{\mu}(s) x}}{\mu+\bar{\mu}(s)} f(\mu(s)) d s \\
& -\frac{b_{2}(\mu) e^{\mu x}}{2} \int_{a}^{b} \frac{\bar{b}_{2}(\mu(s)) e^{\bar{\mu}(s) x}}{\mu+\bar{\mu}(s)} f(\mu(s)) d s .
\end{aligned}
$$

Notice that this expression can be presented as

$$
\mathbb{X}(x) f=b_{1} e^{-\mu x} c_{1}(\mu)+b_{2} e^{\mu x} c_{2}(\mu),
$$

where the functions

$$
\begin{aligned}
& c_{1}(\mu)=-\frac{1}{2} \int_{a}^{b} \frac{\bar{b}_{1}(\mu(s)) e^{-\bar{\mu}(s) x}}{\mu+\bar{\mu}(s)} f(\mu(s)) d s, \\
& c_{2}(\mu)=-\frac{1}{2} \int_{a}^{b} \frac{\bar{b}_{2}(\mu(s)) e^{\bar{\mu}(s) x}}{\mu+\bar{\mu}(s)} f(\mu(s)) d s
\end{aligned}
$$

are analytic functions of $\mu$ in $\mathbb{C} \backslash\left(-\Gamma^{*}\right)$. Notice also that $\Gamma \subseteq \mathbb{C} \backslash$ $\left(-\Gamma^{*}\right)$ by the assumption on $\Gamma$. Thus the functions $c_{1}(\mu), c_{2}(\mu)$ can have only isolated zeros on $\Gamma$ or to be identically zero. 
To simplify a proof that $\mathbb{X}(x)$ is an invertible operator, let us assume that $b_{1}=b_{2}$ and that they are an analytic function of $\mu$. Then the equality $\mathbb{X}(x) f=0$ is equivalent to

$$
\begin{aligned}
& e^{-\mu x} \int_{a}^{b} \frac{\bar{b}_{1}(\mu(s)) e^{-\bar{\mu}(s) x}}{\mu+\bar{\mu}(s)} f(\mu(s)) d s \\
& \quad+e^{\mu x} \int_{a}^{b} \frac{\bar{b}_{1}(\mu(s)) e^{\bar{\mu}(s) x}}{\mu+\bar{\mu}(s)} f(\mu(s)) d s=0 .
\end{aligned}
$$

Taking here $\mu$ around infinity so that $e^{-\mu x}$ is big in absolute value and $e^{\mu x}$ is small we obtain that $\int_{a}^{b}\left(\bar{b}_{1}(\mu(s)) e^{-\bar{\mu}(s) x} /(\mu+\right.$ $\bar{\mu}(s))) f(\mu(s)) d s=0$. Conversely, taking $e^{\mu x}$ big and $e^{-\mu x}$ small the second expression $\int_{a}^{b}\left(\bar{b}_{1}(\mu(s)) e^{\bar{\mu}(s) x} /(\mu+\right.$ $\bar{\mu}(s))) f(\mu(s)) d s=0$ must vanish too. Finally, considering values of $\mu \rightarrow \infty$, we can develop into Taylor series

$$
\frac{1}{\mu+\mu(s)}=\frac{1}{\mu} \sum(-1)^{n} \frac{\mu^{n}(s)}{\mu^{n}}
$$

and plugging it back, the moments of the function $b_{1}(\mu(s)) e^{ \pm \mu(s) x} f(\mu(s))$ must be zero, for all $s$, or that the function $b_{1}(\mu(s)) e^{ \pm \mu(s) x} f(\mu(s))$ is orthogonal to a dense subset $\left\{\mu^{n}(s)\right\}$ of $L^{2}(\Gamma)$. Thus $f(\mu(s))$ is identically zero and $\mathbb{X}(x)$ is invertible for all $x$.

Lemma 11. If $\Gamma$ is a bounded continuous curve, satisfying $\Gamma \cap$ $\left(-\Gamma^{*}\right)=\emptyset$, and the operator $\mathbb{X}(x)$ defined in (57) using analytic functions $b_{1}(\mu)=b_{2}(\mu)$, then $\mathbb{X}(x)$ is bounded and invertible for all $x \in \mathbb{R}$.

Finally, we obtain the following.

Theorem 12. Suppose that $\Gamma$ is a bounded continuous curve, satisfying $\Gamma \cap\left(-\Gamma^{*}\right)=\emptyset$. Define a collection (12)

$$
\mathfrak{V}_{N L S}=\left(A, B(x), \mathbb{X}(x) ; \sigma_{1}, \sigma_{2}, \gamma, \gamma_{*}(x) ; \mathscr{H}, \mathbb{C}^{2}\right),
$$

where $\mathscr{H}=L^{2}(\Gamma), A=2 \mu, \mathbb{X}(x)$, and $B(x)$ are defined by (57) and by (54) for $\left|b_{1}(\mu)\right|^{2}+\left|b_{2}(\mu)\right|^{2} \neq 0$. Then the collection $\mathfrak{V}_{N L S}$ is an NLS regular vessel existing on $I=\mathbb{R}$.

Proof. By the construction the operators are well defined and satisfy the vessel condition. Since the operator $\mathbb{X}(x)$ is invertible, for all $x \in \mathbb{R}$, we obtain that $I=\mathbb{R}$.

3.3. Construction of a Regular NLS Vessel with a Discrete Spectrum. In this section we want to show how to construct a vessel, whose spectrum is a given set of numbers $D=$ $\left\{2 \mu_{n}\right\}$. We define $\mathscr{H}=\ell^{2}$, which is the set of infinite sequences, summable in absolute value. Now we can imitate the construction of the vessel on a curve $\Gamma$ using discretization as follows. We define first the operator $A=\operatorname{diag}\left(2 \mu_{n}\right)$ and for this operator to be bounded, we have to demand that the sequence $D$ be bounded from below and from above as follows.

Definition 13. The sequence $D$ is called bounded if there exists $M>0$ such that $\left|\mu_{n}\right|<M$ for $\mu_{n} \in D$. It is called separated from zero if there exists $m>0$ such that $0<m<\left|\mu_{n}\right|$ for all $\mu_{n} \in D$.

In the next definition we think of $\mathbb{X}(x)$ as an infinite matrix with the entry $m, n$ denoted by $[\mathbb{X}(x)]_{n, m}$

$$
\begin{gathered}
B(x)=\left[\begin{array}{ll}
e^{-\mu_{n} x} b_{1 n} & e^{\mu_{n} x} b_{2 n}
\end{array}\right], \quad\left\{b_{1 n}\right\},\left\{b_{2 n}\right\} \in \mathscr{H}, \\
{[\mathbb{X}(x)]_{n, m}=-\frac{b_{1 n} \bar{b}_{1 m} e^{-\left(\mu_{n}+\bar{\mu}_{m}\right) x}+b_{2 n} \bar{b}_{2 m} e^{\left(\mu_{n}+\bar{\mu}_{m}\right) x}}{2\left(\mu_{n}+\bar{\mu}_{m}\right)} .}
\end{gathered}
$$

We also assume that $[\mathbb{X}(x)]_{n, m}=\left(\left(b_{1 n} \bar{b}_{1 m}-b_{2 n} \bar{b}_{2 m}\right) / 2\right) x$, whenever $\mu_{n}+\bar{\mu}_{m}=0$. The fact that $B(x)$ is a well-defined operator is immediate from the definition. Indeed, since the sequence $\mu_{n}$ is bounded the term $e^{ \pm \mu_{n} x}$ is uniformly bounded in absolute value by $e^{M x}$. The fact that the operator $\mathbb{X}(x)$ is bounded also easily follows from the definitions and from the assumption on $D$ :

$$
\begin{aligned}
\|\mathbb{X}(x)\| & \leq \frac{e^{2 M x}}{2}\left\|\left[\frac{\left|b_{1 n} \bar{b}_{1 m}\right|+\left|b_{2 n} \bar{b}_{2 m}\right|}{\mu_{n}+\bar{\mu}_{m}}\right]\right\| \\
& \leq \frac{e^{2 M x}}{2}\left(\left\|b_{1 n}\right\|^{2}+\left\|b_{2 n}\right\|^{2}\right)<\infty .
\end{aligned}
$$

Under condition that $\mathbb{X}_{0}=\mathbb{X}(0)$ is invertible, there exists a nontrivial interval (of length at least $1 /\left\|\mathbb{X}_{0}^{-1}\right\|$ ) on which $\mathbb{X}(x)$ is invertible too. Thus we obtain the following theorem.

Theorem 14. Suppose that we are given a bounded, separated from zero set $\left\{\mu_{n}\right\}$, and two $\ell^{2}$ sequences $\left\{b_{1 n}\right\},\left\{b_{2 n}\right\}$. Define a collection

$$
\mathfrak{V}_{N L S}=\left(A, B(x), \mathbb{X}(x) ; \sigma_{1}, \sigma_{2}, \gamma, \gamma_{*}(x) ; \mathscr{H}, \mathbb{C}^{2}\right),
$$

where $\mathscr{H}=\ell^{2}, A=\operatorname{diag}\left(2 \mu_{n}\right), \mathbb{X}(x)$, and $B(x)$ are defined by (63) for $\left|b_{1 n}\right|+\left|b_{2 n}\right| \neq 0$. Suppose that the operator $\mathbb{X}(0)$ is invertible. Then the collection $\mathfrak{V}_{N L S}$ is an NLS regular vessel existing on a nontrivial interval I including zero of length at least $1 /\left\|\mathbb{X}_{0}^{-1}\right\|$.

\section{Evolutionary Regular NLS Vessel}

We present a construction of solutions of (1) which has initial value $\beta(x, 0)$ arising from a regular NLS vessel. For this we will insert dependence on the variable $t$ into the vessel operators and postulate evolution of the operators $B, \mathbb{X}$ with respect to $t$. This is done in the next definition.

Definition 15. The collection

$$
\mathfrak{V}_{\mathrm{ENLS}}=\left(A, B(x, t), \mathbb{X}(x, t) ; \sigma_{1}, \sigma_{2}, \gamma, \gamma_{*}(x) ; \mathscr{H}, \mathbb{C}^{2}\right)
$$

is called evolutionary regular NLS vessel if additionally to (13), (14), (15), and (16) the bounded operators satisfy also the equations

$$
\begin{gathered}
\frac{\partial}{\partial t} B(x, t)=i A \frac{\partial}{\partial x} B(x, t), \\
\frac{\partial}{\partial t} \mathbb{X}=i A B \sigma_{2} B^{*}-i B \sigma_{2} B^{*} A^{*} .
\end{gathered}
$$


Theorem 16. Moments $H_{N}(x, t)$ of the vessel $\mathfrak{V}_{E N L S}$ satisfy the following recurrence equation:

$$
\left(H_{N}\right)_{t}^{\prime}=i\left(H_{N+1}\right)_{x}^{\prime}+i\left(H_{0}\right)_{x}^{\prime} \sigma_{1} H_{N}
$$

And the transfer functions satisfy

$$
S_{t}^{\prime}=i \lambda S_{x}^{\prime}+i\left(H_{0}\right)_{x}^{\prime} \sigma_{1} S
$$

Proof. We will calculate the derivative of $H_{N}$ using the vessel conditions:

$$
\begin{aligned}
& \left(H_{N}\right)_{t}^{\prime}=\left(B^{*} \mathbb{X}^{-1} A^{N} B\right)_{t}^{\prime} \\
& =\left(B^{*}\right)_{t}^{\prime} \mathbb{X}^{-1} A^{N} B+B^{*}\left(\mathbb{X}^{-1}\right)_{t}^{\prime} A^{N} B+B^{*} \mathbb{X}^{-1} A^{N}(B)_{t}^{\prime} \\
& =\text { using (67), (68) } \\
& =\left(B^{*}\right)_{x}^{\prime}\left(-i A^{*}\right) \mathbb{X}^{-1} A^{N} B \\
& -B^{*} \mathbb{X}^{-1}\left(i A B \sigma_{2} B^{*}-i B \sigma_{2} B^{*} A^{*}\right) \mathbb{X}^{-1} A^{N} B \\
& +B^{*} \mathbb{X}^{-1} A^{N}(i A) B_{x}^{\prime} \\
& \text { = using (14) } \\
& =i\left(B^{*}\right)_{x}^{\prime}\left(\mathbb{X}^{-1} A+\mathbb{X}^{-1} B \sigma_{1} B^{*} \mathbb{X}^{-1}\right) A^{N} B \\
& -i B^{*} \mathbb{X}^{-1} A B \sigma_{2} B^{*} \mathbb{X}^{-1} A^{N} B \\
& -i B^{*} \mathbb{X}^{-1} B \sigma_{2} B^{*}\left(\mathbb{X}^{-1} A+\mathbb{X}^{-1} B \sigma_{1} B^{*} \mathbb{X}^{-1}\right) A^{N} B \\
& +i B^{*} \mathbb{X}^{-1} A^{N+1} B_{x}^{\prime} \\
& =i\left[\left(B^{*}\right)_{x}^{\prime} \mathbb{X}^{-1} A^{N+1} B-B^{*} \mathbb{X}^{-1} A B \sigma_{2} B^{*} \mathbb{X}^{-1} A^{N+1} B\right. \\
& \left.+i B^{*} \mathbb{X}^{-1} A^{N+1} B_{x}^{\prime}\right] \\
& +i\left(B^{*}\right)_{x}^{\prime} \mathbb{X}^{-1} B \sigma_{1} B^{*} \mathbb{X}^{-1} A^{N} B \\
& -i B^{*} \mathbb{X}^{-1} A B \sigma_{2} B^{*} \mathbb{X}^{-1} A^{N} B \\
& -i B^{*} \mathbb{X}^{-1} B \sigma_{2} B^{*} \mathbb{X}^{-1} B \sigma_{1} B^{*} \mathbb{X}^{-1} A^{N} B \\
& \text { = using (15) and the definition of moments } \\
& =i\left(H_{N+1}\right)_{x}^{\prime}+i\left(B^{*}\right)_{x}^{\prime} \mathbb{X}^{-1} B \sigma_{1} H_{N} \\
& +B^{*} \mathbb{X}^{-1}(B)_{x}^{\prime} \sigma_{1} H_{N}+B^{*}\left(\mathbb{X}^{-1}\right)_{x}^{\prime} B \sigma_{1} H_{N} \\
& =i\left(H_{N+1}\right)_{x}^{\prime}+i\left(H_{0}\right)_{x}^{\prime} \sigma_{1} H_{N}
\end{aligned}
$$

The formula for $S_{t}^{\prime}$ is an immediate consequence of the series representation of $S$ using moments and equating the powers of $\lambda$.

Theorem 17. Suppose that $\mathfrak{V}_{\text {ENLS }}$ is an evolutionary NLS vessel; then, $\beta(x, t)(20)$ satisfies the evolutionary NLS equation (1).
Proof. Consider the equality of mixed derivatives

$$
\frac{\partial}{\partial x} \frac{\partial}{\partial t} S=\frac{\partial}{\partial t} \frac{\partial}{\partial x} S
$$

Plugging (26) and (70) here and representing $S$ as a series of moments, we can further consider both sides of this equality and make the corresponding coefficients of $\lambda^{-n}$ equal

$$
\begin{aligned}
\frac{\partial}{\partial x}\left[i \lambda S_{x}^{\prime}+i\left(H_{0}\right)_{x}^{\prime} \sigma_{1} S\right] \\
\quad=\frac{\partial}{\partial t}\left[\sigma_{1}^{-1}\left(\sigma_{2} \lambda+\gamma_{*}\right) S-S \sigma_{1}^{-1}\left(\sigma_{2} \lambda+\gamma\right)\right] .
\end{aligned}
$$

For example, taking the free coefficient, we wil obtain that

$$
\left(\gamma_{*}\right)_{t}^{\prime}=-i \gamma_{*}\left(H_{0}\right)_{x}^{\prime} \sigma_{1}+i \sigma_{1}\left(H_{0}\right)_{x x}^{\prime \prime} \sigma_{1}+i \sigma_{1}\left(H_{0}\right)_{x}^{\prime} \gamma_{*} .
$$

Finally, from linkage condition (16)

$$
\gamma_{*}=\left[\begin{array}{cc}
0 & \beta(x, t) \\
-\beta^{*}(x, t) & 0
\end{array}\right]
$$

and using the formula for $\left(H_{0}\right)_{x}^{\prime}=\left[\begin{array}{cc}|\beta|^{2} & (\beta)_{x}^{\prime} \\ \left(\beta^{*}\right)_{x}^{\prime} & -|\beta|^{2}\end{array}\right]$ we will obtain that the entry 12 is translated into (1) for $\beta(x, t)$, defined by (20).

So in order to solve (1) with initial $\beta(x, 0)$ which arises from a vessel, it is enough to add dependence on $t$ so that $B(x, 0)=B(x), \mathbb{X}(x, 0)=\mathbb{X}(x)$, and the differential equations (67) and (68) hold. We will show that it is a simple task in the next examples.

\section{Examples of Constructions of Solutions of the Evolutionary NLS Equation}

We present examples of solutions of the evolutionary NLS (1) when the initial value for $t=0, \beta(x)$ is analytic on $\mathbb{R}$ and arises from a regular NLS vessel. We show how to construct the evolutionary vessel, coinciding with the vessel realizing $\beta(x)$ for $t=0$. Then the beta function of this evolutionary vessel is a solution of (1) with the initial value.

5.1. Construction of a Solution for Evolutionary NLS Vessel from a Realized Function. Suppose that $\beta(x)$ was constructed from a realized function as in Section 3.1

$$
S\left(\lambda, x_{0}\right)=I-B_{0}^{*} \mathbb{X}_{0}^{-1}(\lambda I-A)^{-1} B_{0} .
$$

Then the construction can proceed the following steps, each one requiring a solution of linear differential equation with initial value. Construct $B(x)$ and $\mathbb{X}(x)$ by formulas (49) and (50). Then solve

$$
\begin{gathered}
\frac{\partial}{\partial t} B(x, t)=i A \frac{\partial}{\partial x} B(x, t)=i A\left(-A B(x, t) \sigma_{2}\right), \\
B(x, 0)=B(x) .
\end{gathered}
$$


Finally, define

$$
\begin{aligned}
\mathbb{X}(x, t)= & \mathbb{X}(x) \\
& +\int_{0}^{t}\left[i A B(x, s) \sigma_{2} B^{*}(x, s)\right. \\
& \left.\quad-i B(x, s) \sigma_{2} B^{*}(x, s) A^{*}\right] d s .
\end{aligned}
$$

All the vessel equations will be satisfied by the construction and can be easily verified.

5.2. Solution of the Evolutionary NLS with the Spectrum on a Curve $\Gamma$. If we are given $\beta(x)$ which arises from a vessel on a curve, we can solve explicitly equations for $B(x, t)$ and $\mathbb{X}(x, t)$ as follows:

$$
\begin{array}{r}
B(x)=\left[\exp \left(-\mu x-2 i \mu^{2} t\right) b_{1}(\mu) \exp \left(\mu x+2 i \mu^{2} t\right) b_{2}(\mu)\right], \\
b_{1}(\mu), b_{2}(\mu) \in \mathscr{H} .
\end{array}
$$

This function coincides with $B(x)$ for $t=0$ and satisfies (13) and (67). The formula for $\mathbb{X}(x, t)$ is as follows:

$$
\begin{aligned}
& (\mathbb{X}(x) f)(\mu) \\
& =-\frac{1}{2} \int_{a}^{b}\left(\left(b_{1}(\mu) \bar{b}_{1}(\mu(s))\right.\right. \\
& \left.\quad \times \exp \left(-(\mu+\bar{\mu}(s)) x-i\left(\mu^{2}+\bar{\mu}^{2}(s)\right) t\right)\right) \\
& \left.\quad \times(\mu+\bar{\mu}(s))^{-1}\right) f(\mu(s)) d s \\
& -\frac{1}{2} \int_{a}^{b}\left(\left(b_{2}(\mu) \bar{b}_{2}(\mu(s))\right.\right. \\
& \left.\quad \times \exp \left((\mu+\bar{\mu}(s)) x+i\left(\mu^{2}+\mu^{2}(s)\right) t\right)\right) \\
& \left.\quad \times(\mu+\bar{\mu}(s))^{-1}\right) f(\mu(s)) d s .
\end{aligned}
$$

This constructs a solution of the (1) with initial $\beta(x)$ arising from an NLS vessel with the spectrum on a curve $\Gamma$.

5.3. Solution of the Evolutionary NLS with the Spectrum on a Discrete Set. Similarly to the continuous spectrum case we define

$$
\begin{array}{r}
B(x)=\left[\exp \left(-\mu_{n} x-2 i \mu_{n}^{2} t\right) b_{1 n} \exp \left(\mu_{n} x+2 i \mu_{n}^{2}\right) b_{2 n}\right], \\
\left\{b_{1 n}\right\},\left\{b_{2 n}\right\} \in \mathscr{H},
\end{array}
$$

$$
\begin{aligned}
{[X(x)]_{n, m}=} & -\left(b_{1 n} \bar{b}_{1 m} e^{-\left(\mu_{n}+\bar{\mu}_{m}\right) x-2 i\left(\mu_{n}^{2}+\bar{\mu}_{m}^{2}\right) t}\right. \\
& \left.+b_{2 n} \bar{b}_{2 m} e^{\left(\mu_{n}+\bar{\mu}_{m}\right) x+2 i\left(\mu_{n}^{2}+\bar{\mu}_{m}^{2}\right) t}\right) \\
& \times\left(2\left(\mu_{n}+\bar{\mu}_{m}\right)\right)^{-1} .
\end{aligned}
$$

We also assume that $[\mathbb{X}(x)]_{n, m}=\left(\left(b_{1 n} \bar{b}_{1 m}-b_{2 n} \bar{b}_{2 m}\right) / 2\right)(x+$ $\left.2 i \mu_{n} t\right)$, whenever $\mu_{n}+\bar{\mu}_{m}=0$.
5.4. Solitons. We can also consider finite dimensional case: $\operatorname{dim} \mathscr{H}<\infty$. Suppose that $\mathscr{H}=\mathbb{C}^{N}$ and fixing nonzero values $\mu_{1}, \mu_{2}, \ldots, \mu_{N}$, satisfying $\mu_{i}+\bar{\mu}_{j} \neq 0$, for all $1 \leq i, j \leq N$, we will obtain that

$$
B(x)=\left[\begin{array}{cc}
\exp \left(-\mu_{1} x-2 i \mu_{1}^{2} t\right) b_{11} & \exp \left(\mu_{1} x+2 i \mu_{1}^{2}\right) b_{21} \\
\exp \left(-\mu_{2} x-2 i \mu_{2}^{2} t\right) b_{12} & \exp \left(\mu_{1} x+2 i \mu_{2}^{2}\right) b_{22} \\
\vdots & \vdots \\
\exp \left(-\mu_{N} x-2 i \mu_{N}^{2} t\right) b_{1 N} & \exp \left(\mu_{N} x+2 i \mu_{N}^{2}\right) b_{2 N}
\end{array}\right]
$$

and the operator $\mathbb{X}(x)$ is $N \times N$ matrix, defined by formula (82). Then the function

$$
\beta(x, t)=\left[\begin{array}{ll}
1 & 0
\end{array}\right] B^{*}(x) \mathbb{X}^{-1}(x) B(x)\left[\begin{array}{l}
0 \\
1
\end{array}\right]
$$

is a Soliton solution of (1), because it is constructed from pure exponents.

\section{Conflict of Interests}

The author declares that there is no conflict of interests regarding the publication of this paper.

\section{References}

[1] V. E. Zacharov and A. B. Shabath, "Integration of the nonlinear equations of mathematical physics by the method of the inverse scattering problem," Funktsional. Anal. i Prilozhen, vol. 8, no. 3, pp. 43-53, 1974 (Russian), translation in Functional Analysis and Its Applications, vol. 8, no. 3, pp. 226-235, 1974.

[2] A. Melnikov, "On a theory of vessels and the inverse scattering," In press, http://arxiv.org/abs/1103.2392.

[3] A. Melnikov, "Solution of the KdV equation on the line with analytic initial potential," Accepted to Journal of Mathematical Physics, http://arxiv.org/abs/1303.5324.

[4] J. A. Ball, I. Gohberg, and L. Rodman, Interpolation of Rational Matrix Functions, vol. 45 of Operator Theory: Advances and Applications, Birkhäuser, Basel, Switzerland, 1990.

[5] A. Melnikov, "Finite-dimensional Sturm-Liouville vessels and their tau functions," Integral Equations and Operator Theory, vol. 71, no. 4, pp. 455-490, 2011.

[6] D. Alpay, A. Melnikov, and V. Vinnikov, "Un algorithme de Schur pour les fonctions de transfert des systèmes surdéterminés invariants dans une direction," Comptes-Rendus Mathématiques, vol. 347, no. 13-14, pp. 729-733, 2009.

[7] D. Alpay, A. Melnikov, and V. Vinnikov, "Schur algorithm in the class $S I$ of $J$-contractive functions intertwining solutions of linear differential equations," Integral Equations and Operator Theory, vol. 74, no. 3, pp. 313-344, 2012.

[8] A. Melnikov and V. Vinnikov, "Null/pole interpolation problem in the class RI of rational functions intertwining solutions of linear differential equations," Complex Analysis and Operator Theory, 2014.

[9] A. Melnikov, "Solution of the KdV equation using evolutionary vessels," submitted, http://arxiv.org/abs/1110.3495.

[10] A. Melnikov, "Inverse scattering of canonical systems and their evolution," Complex Analysis and Operator Theory, 2014. 
[11] M. S. Livšic, "Commuting nonselfadjoint operators and solutions of systems of partial differential equations generated by them," Soobshch. Akad. Nauk Gruzin. SSR, vol. 91, no. 2, pp. 281284, 1978 (Russian).

[12] M. S. Livšic, "Vortices of 2D systems," Operator Theory: Advances and Applications, vol. 123, pp. 7-41, 2001.

[13] A. Melnikov and V. Vinnikov, "Overdetermined 2D systems invariant in one direction and their transfer functions," submitted, http://arXiv.org/abs/0812.3779.

[14] A. Melnikov and V. Vinnikov, "Overdetermined conservative 2D systems, invariant in one direction and a generalization of Potapov's theorem," http://arxiv.org/abs/0812.3970.

[15] I. Gohberg and M. Krein, Introduction to the Theory of Linear Non-Selfadjoint Operators, Translations of AMS, 1969. 


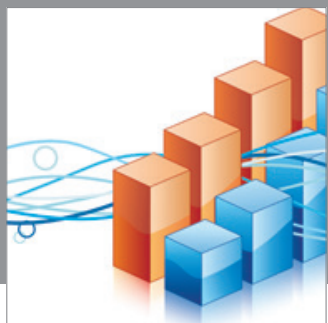

Advances in

Operations Research

mansans

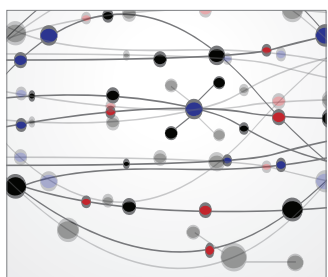

The Scientific World Journal
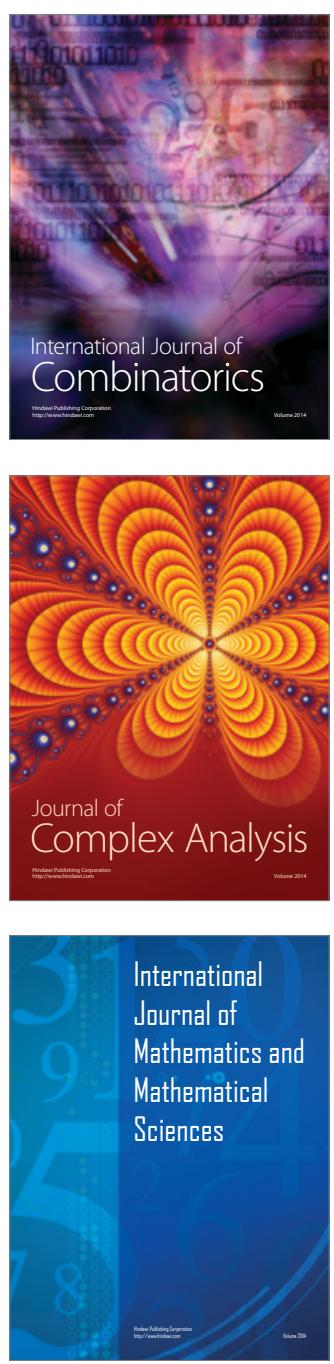
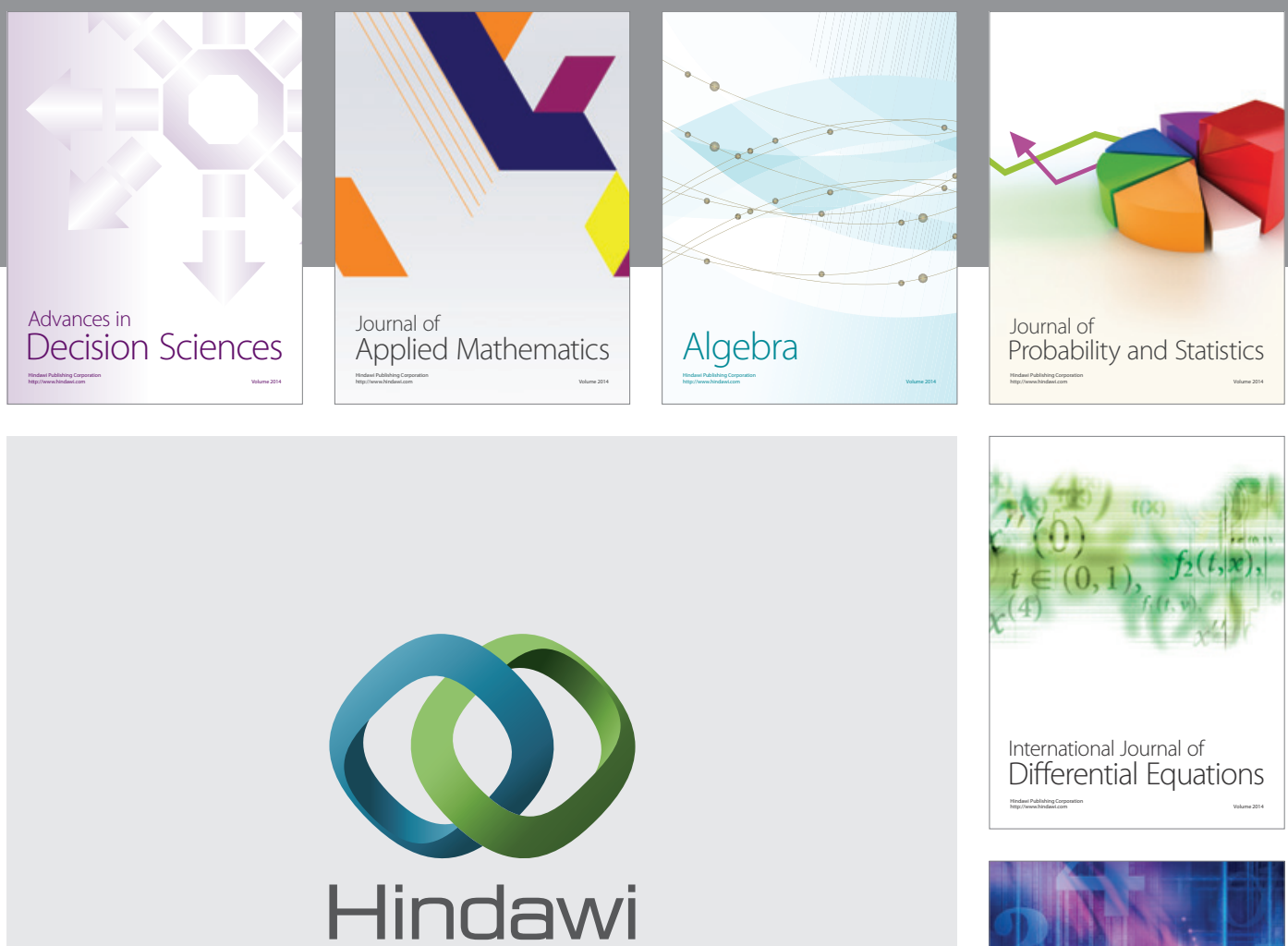

Submit your manuscripts at http://www.hindawi.com
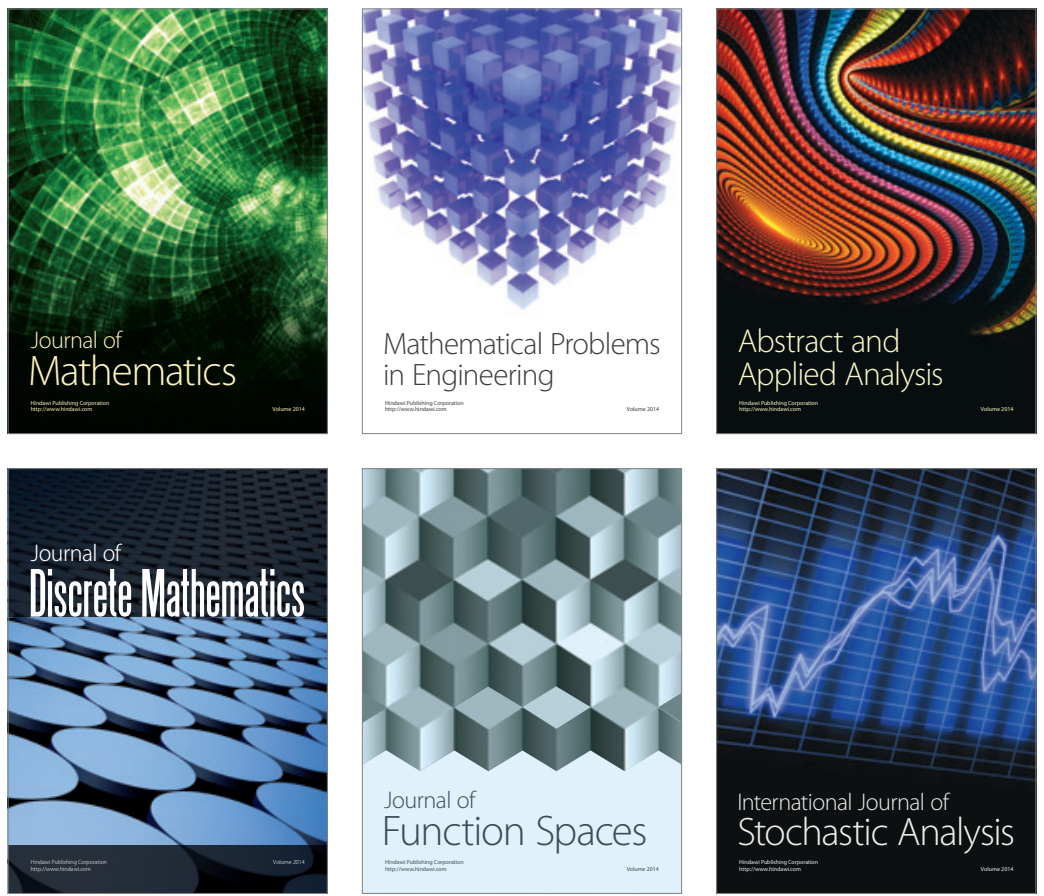

Journal of

Function Spaces

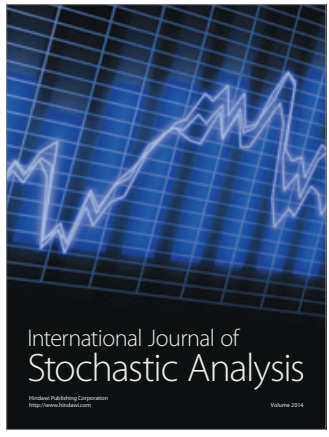

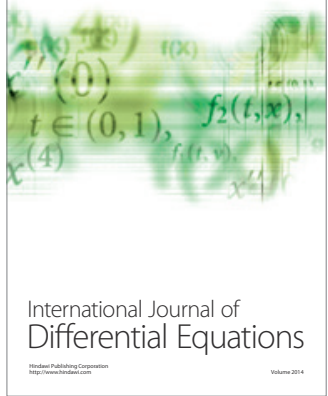
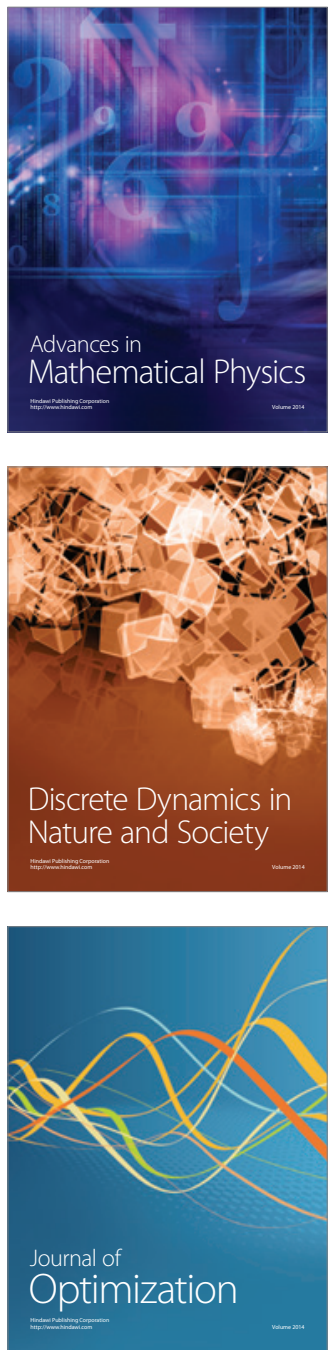